\title{
Microgels and apparatus for PAGE of nucleic acids in one or two dimensions
}

\author{
Hans G Thormar',3, Gudmundur H Gunnarsson ${ }^{1,3}$, Bjarki Gudmundsson 1,2,3, Kristjan Leosson4, \\ Peter Estibeiro 3 \& Jon J Jonsson ${ }^{\star, 1,2}$ \\ ${ }^{1}$ Department of Biochemistry \& Molecular Biology, Faculty of Medicine, University of Iceland, Reykjavik, \\ Iceland; 'Department of Genetics \& Molecular Medicine, Landspitali - National University Hospital, \\ Reykjavík, Iceland; ' $L i f e i n d ~ e h f$, Reykjavik, Iceland; ' ${ }^{4}$ epartment of Materials Science, Biotechnology \& \\ Energy, Innovation Center, Reykjavik, Iceland
}

BioTechniques 65: 15-19 (July 2018) 10.2144/btn-2018-0030

Keywords: apparatus $\bullet$ DNA $\bullet$ electrophoresis $\bullet$ microgels $\bullet$ nucleic acids $\bullet$ one-dimension $\bullet$ PAGE $\bullet$ RNA $\bullet$ two-dimensions

We describe a system for horizontal 1D or 2D PAGE comprising an apparatus and microgels. There is no buffer outside the gel, making handling and sample loading easy. Specially designed electrodes on all four sides allow 2D electrophoresis without gel rotation. Electrophoresis is completed within 20 min and sensitivity is in the subnanogram range. The system is temperature controlled for speed, denaturation of nucleic acid molecules and maintaining molecules single-stranded. The system allows characterization of structure, conformation and damage in complex nucleic acid preparations. Besides quick 1D PAGE, 2D applications include characterization of efficiency of complex molecular procedures, checking quality of biosamples and detecting DNA damage in cells and body fluids. The system should also run protein gels.

\section{Introduction}

PAGE is a common method for analyzing or isolating biomolecules. Despite advances in capillary electrophoresis, micro- and nano-fluidics and in liquid-phase microextractions [1-3], the minigel apparatus is still the choice of many investigators. Although PAGE equipment has been improved over the years, the methodology remains similar, as are the gel matrices, loading and running buffers. Nucleic acids have been labelled in different ways and are now typically detected with fluorescent dyes [4-6].

In PAGE, for a given magnitude of external electric field, the migration velocity of molecules is dependent on their mass, charge and conformation. The pore size of the gel matrix determines the separation based on size and shape. Labeling prior to electrophoresis or post-staining of the gels are used to detect molecules. Molecular markers are run in parallel to the sample for assessment of molecular mass. Electrophoresis can be used to separate molecules by properties other than their mass; for example, by treatment of the gel matrix before or during electrophoresis. For example, a pH gradient can be formed across the gel matrix prior to electrophoresis and molecules migrate in the gel matrix until their overall charge is zero (isoelectric point) [7].

To separate molecules within a sample based on different properties, 2D electrophoresis can be used. The sample is subjected to electrophoresis in one direction and the second dimension is perpendicular to the first. The gel matrix can have different properties in the first and the second direction or the gel matrix is treated between dimensions. Examples include treatment where molecules are chemically modified or denatured with heat. An example of the latter is $2 \mathrm{D}$ strandness-dependent electrophoresis (2D-SDE), a method to analyze the composition of complex mixtures of ssDNA and dsDNA [8,9]. In the first dimension, molecules are separated based on their conformation, strandness (i.e., ssDNA and dsDNA), and base pair or nucleotide length. After the first dimension, the molecules are denatured and separated only by nucleotide length in the second dimension.

Another electrophoresis method that is based on modifying molecular properties is the combined single-stranded conformational polymorphism/heteroduplex analysis (SSCP/HA), which is based on effective separation of the doublestranded homo- or hetero-duplexes from single-stranded molecules [10]. Denaturing gradient gel electrophoresis (DGGE) and temperature-gradient gel electrophoresis (TGGE) are also examples of methods that both rely on the transition of dsDNA fragments into ssDNA fragments during the electrophoresis [11,12]. The transition is facilitated by the denaturing gradient

\section{METHOD SUMMARY}

We developed a microgel apparatus for 1D and 2D PAGE. The platform needs no external buffers since there is enough buffer capacity within the gel matrix. The platform is temperature controlled and has electrodes on all four sides so that the gel does not have to be rotated between dimensions. The system can be used in diverse applications including 1D PAGE and 2D characterization of size, structure, conformation and damage in complex nucleic acid samples. 
created with either a chemical composition gradient or a temperature increase. Such methods have been used to analyze the melting profile of DNA fragments both in 1D and 2D setups.

One way of carrying out 2D gel electrophoresis is to run the electrophoresis in the first dimension and then physically rotate the gel $90^{\circ}$, enabling electrophoresis to be carried out in the second dimension. This can be done manually or using a robotic system. An alternative setup would be an apparatus with two sets of opposed electrodes where the gel would not have to be rotated. In this configuration the pair of electrodes not in use could potentially distort the electrical field generated by the other pair of electrodes through shortcut. This would affect the distribution of molecules after electrophoresis and reduce the area of the gel where 2D gel electrophoresis can be carried out.

The electrophoresis system introduced here is a novel 2D electrophoresis system for PAGE in which a uniform electric field can be produced across the electrophoresis gel in both dimensions to provide an even distribution of separated molecules and to maximize the useful area of the electrophoresis gel. The system is based on a microgel format in a closed single-use gel cassette with internal electrodes on all four sides eliminating the need to rotate the gel between dimensions. The user can load samples directly to the wells without loading buffers and control the temper- ature of the gel during the electrophoresis to allow different kinds of separation in the two dimensions. Compared to conventional PAGE, this format allows users to perform very quick and robust gel electrophoresis with minimal skills and hands-on time. Longer runs up to at least 20 min are possible in both directions, allowing better separation of larger molecules. Like PAGE in general, gel fragments containing molecules of interest can be cut out of the gel after electrophoresis and nucleic acids isolated for various downstream applications. The resolution is similar to PAGE minigels and, compared with agarose, better for smaller fragments and worse for larger kilobase-sized fragments. Because of the small electrophoresis area used for each sample, the minimal amount of nucleic acids that can be analyzed is in the subnanogram range. This platform could be useful for characterization of the efficiency of molecular procedures with complex samples such as optimizations of library preparations for NGS and genomic amplifications. This platform can also be used to characterize quality of and damage to DNA samples in biological specimens such as FFPE samples, ChIP-Seq samples and liquid biopsies.

\section{Material \& methods}

\section{Electric field calculation}

The electric field distribution for different electrode configurations was simulated
(A)

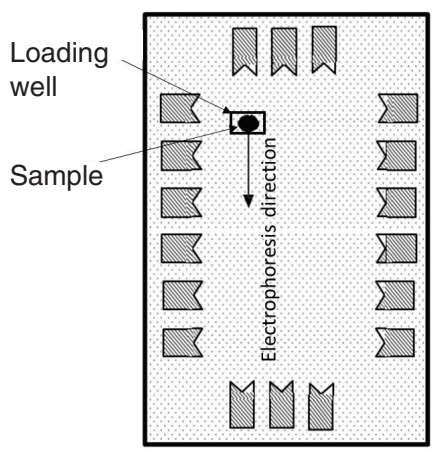

(B)

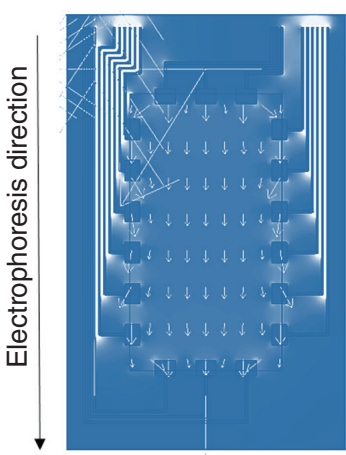

(C)

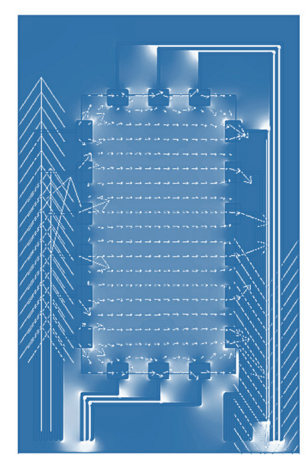

Electrophoresis direction
Figure 1. Graphical illustrations of electric fields and current density calculations on the horizontal credit card-sized microgel cassette. First dimension electrophoresis is carried out from top to bottom and the second dimension electrophoresis from left to right. (A) Schematic drawing of electrode configuration shown as 3 top to bottom and 6 left to right and electrodes in the figure [13]. The sample (black dot) is loaded into the rectangular loading well. (B) Simulated electrophoresis in the first dimension (down in the figure). The arrows indicate the flow of the current. (C) Simulated electrophoresis in the second dimension (to the right in the figure). A strong electric field was predicted between the closely spaced electrode wires in the passive direction. In the final version of the electrode connections, the electrode arrangement was the same, but the connection wires for each electrode were separated. using the finite-element software COMSOL (COMSOL, Inc., MA, USA).

\section{Gel cassette}

The cassette was made using acrylic plates (Goodfellow Cambridge Limited, Huntingdon, UK), where the bottom plate was $0.5-\mathrm{mm}$ thick, the middle plate spacer was $2.5-\mathrm{mm}$ thick and the top plate was 1.0-mm thick. Electrodes were printed on the bottom plate using liquid carbon paste (Electra Polymers Ltd, Kent, UK).

Instrument electrode connection unit The instrument electrode connection unit was made with spring-loaded pogo pins connected via a circuit board to a relay that opens/closes the circuit depending on the direction of the electrophoresis (Wideblue Ltd, Glasgow, UK). A regular power supply (capable of constant current and constant power) was connected to the circuit board to perform the electrophoresis.

\section{Thermal control}

The gel/cassette temperature was controlled using Peltier element (CP-036), temperature control unit (TC-36-25-RS485) and power supply (PS-12-8.4A) from TE Technology, Inc. (MI, USA).

\section{Gel electrophoresis}

The gel solution typically consisted of 4-6\% acrylamide mixed with $1.25 \times \mathrm{TBE}$ buffer. If necessary, urea was mixed in the gel to a final concentration of $7 \%$. The electrophoresis was performed using the following conditions: first dimension: gel cassette was cooled down to $4^{\circ} \mathrm{C}$. Nucleic acid sample (1-6 $\mu$ l) was loaded in each well along with a $100 \mathrm{bp}$ size marker (Fermentas/Thermo-Fisher, MA, USA). The gel was run at $200 \mathrm{~V}$, constant $36 \mathrm{~mA}$ for 5-13 min depending on the application. Before the second dimension the gel was heated to $85^{\circ} \mathrm{C}$ for $2 \mathrm{~min}$, then the temperature was set to $55^{\circ} \mathrm{C}$ for $3 \mathrm{~min}$ before second dimension electrophoresis at $55^{\circ} \mathrm{C}$ and run at $60 \mathrm{~V}$, constant $36 \mathrm{~mA}$ for 4-7 $\mathrm{min}$.

\section{Gel staining \& analysis}

The gel cassette was opened, and the gel immersed in fluorescent staining solution containing $5 \mu \mathrm{l}$ 10.000x Ribogreen (Thermo-Fisher) dye and $100 \mathrm{ml} \mathrm{H} \mathrm{H}_{2} \mathrm{O}$ for $10 \mathrm{~min}$. If the amount of nucleic acid is very low, the amount of Ribogreen dye can be increased. Thereafter the gel was washed 


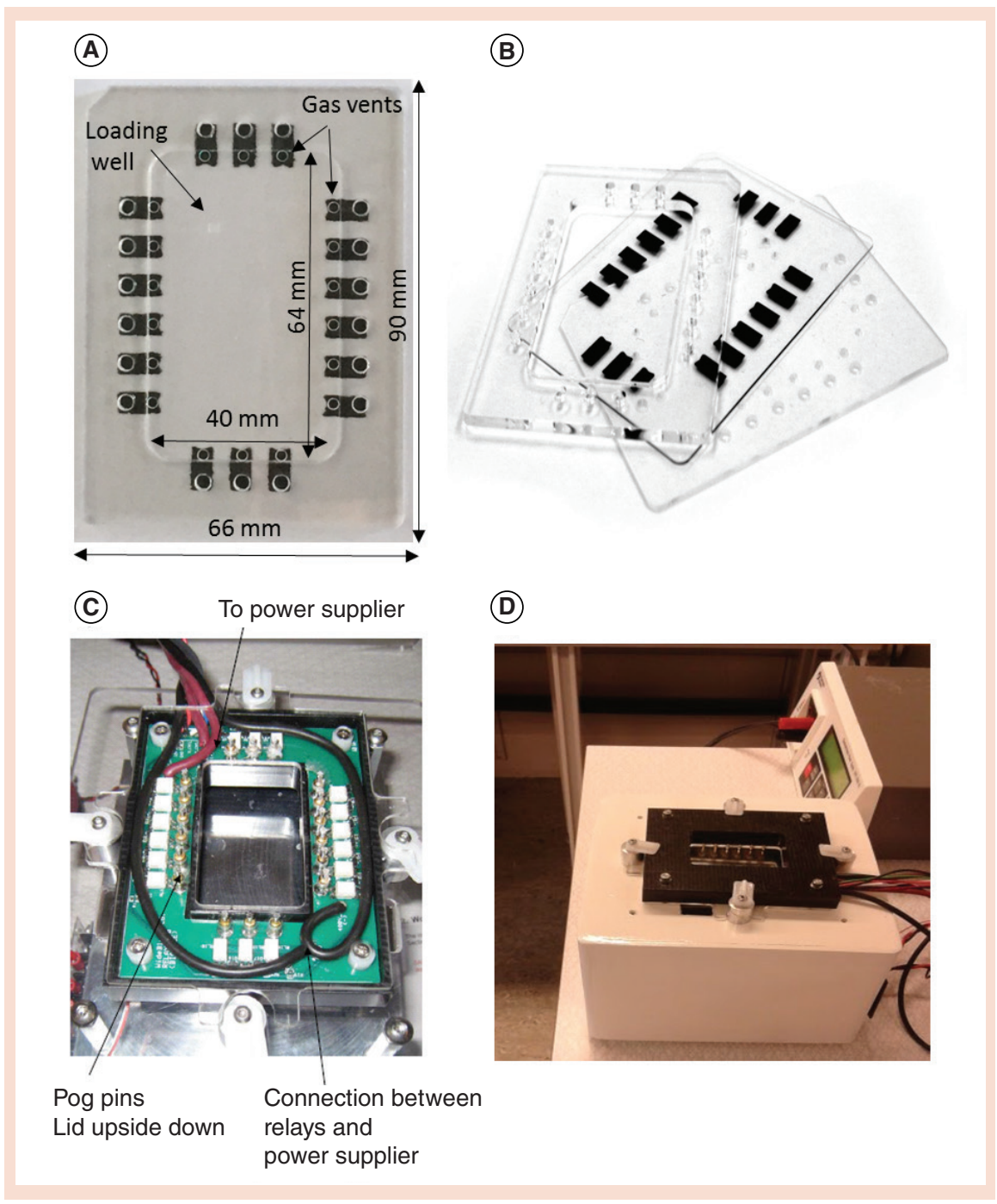

Figure 2. Apparatus and microgels. (A) Shape of electrodes, boundaries of the gel and location of vents to allow easy elimination of gas molecules. Dimensions of the gel card and gel are shown. (B) The three layers of the gel cassette. (C) Apparatus lid, with electric hardwire and pogo pins for connection to the gel electrodes. (D) Apparatus with power supplier in the background.

with $\mathrm{H}_{2} \mathrm{O}$, and imaged in a fluorescent scanner (Typhoon 8610, GE Healthcare, WI, USA). The gel image was analyzed, and DNA fraction quantified using ImageQuant ${ }^{\text {TM }}$ software.

\section{Results \& discussion}

We designed a microgel platform for PAGE that can be used to perform 1D or 2D electrophoresis of nucleic acids in a closed gel cassette. We simulated the electrophoresis and calculated the electric field distribution using COMSOL, a finiteelement software. After conducting a series of simulations, using different sizes of gel cassettes and different electrode configurations we fabricated gel cassettes with an optimized design, where electrodes of a certain size are evenly separated around the edges of the gel (Figure 1). This configuration resulted in the largest area of evenly distributed electric field across the gel both in the first and second dimension. Specially designed electrodes on all four sides allow 2D electrophoresis without removing and turning the gel in between dimensions.

To perform PAGE in a quick and costeffective manner, the shape and material of the gel cassette was carefully considered. Different constructs and materials were tested, including plastics, glass and pure acrylic for transparency and heat transfer efficiency. For the bottom plate casted $0.5 \mathrm{~mm}$ thick acrylic was used as it is thin enough to tolerate and transfer heat efficiently and is also transparent enough to allow visualization of molecules excited using fluorescent molecular dyes. Thicker bottom plates resulted in less efficient separation of molecules. Additionally, acrylic gel cassettes are low cost and have sufficient mechanical strength at the thickness allowing rapid heat conductance. The gel cassette easily tolerates temperature changes from $0^{\circ} \mathrm{C}$ to $85^{\circ} \mathrm{C}$ without distortion, which is a very important factor for 2D applications based on variation in temperature; for example, TGGE and 2D-SDE. The system is temperature controlled. For 1D electrophoresis, cooling of the gel allows the user to perform electrophoresis very quickly without heating the gel matrix. Elevated temperature is used to denature nucleic acid molecules between dimensions. The second dimension electrophoresis is run under elevated temperature conditions where single-stranded nucleic acid molecules have no secondary structure and migrate only according to nucleotide length. This system allows straightforward characterization of structure, conformation and damage in complex nucleic acid preparations.

Using a closed gel system for electrophoresis with no additional running buffers can lead to depletion of ions because of insufficient buffer capacity. Buffer concentration calculations were performed to estimate the optimum gel thickness and buffer concentration, to avoid reduction of buffer capacity affecting migration. These calculations indicated that regular PAGE gel of normal thickness $(1.0 \mathrm{~mm})$ would not suffice under these conditions. This led us to test between 2 to $4 \mathrm{~mm}$-thick middle spacer plates (controlling the thickness of the gel) and a buffer of 1.0, 1.25 and 1.5 $\times$ Tris Borate EDTA (TBE). After a series of electrophoresis experiments using different buffer concentrations and gel thickness we found that $1.25 \times$ TBE and a gel thickness of $2.5 \mathrm{~mm}$ resulted in efficient electrophoresis with good separation of bands.

Correct material for the electrodes is important. We tested platinum and gold electrodes, silver paste, silver epoxy, aluminum, brass, copper and carbon paste. At the cathode, highly reactive $\mathrm{H}_{2}$ is released during electrophoresis. Platinum, gold and carbon paste were all found to be resistant to $\mathrm{H}_{2}$. In the final version, the electrodes were made from carbon paste since they are inexpensive and easy to print onto the acrylic bottom plate.

During electrophoresis, $\mathrm{H}_{2}$ and $\mathrm{O}_{2}$ form at the cathode and anode, respectively. To avoid build-up of such gases within the gel cassette, we designed gas vents above the tip of each electrode (Figure 2). 
(A)

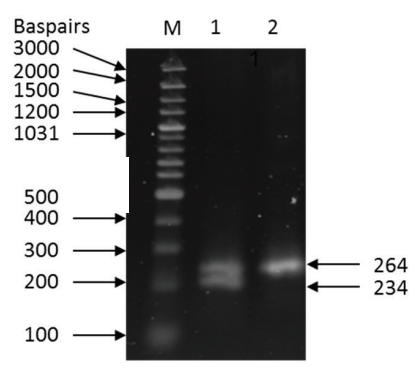

(C)

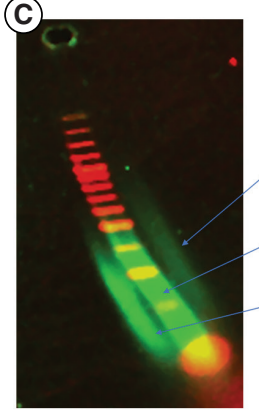

(B)

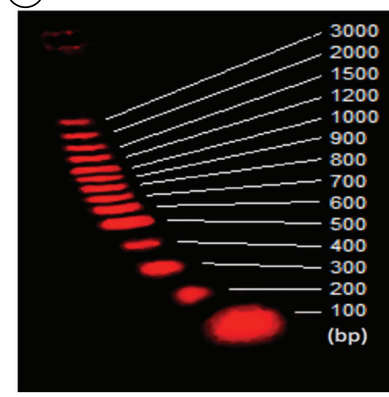

(D)

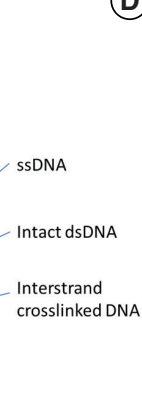

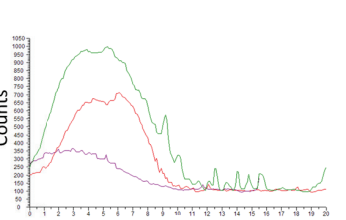

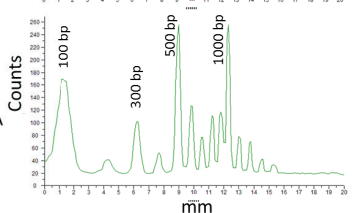

(E)

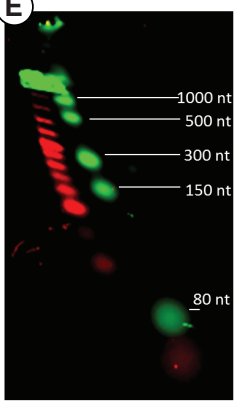

Figure 3. Examples of gel electrophoresis using the microgel apparatus. (A) Conventional 1D electrophoresis of a 100 bp size marker and separation of two bands of 234 and 264 bp. (B) 2D electrophoresis of the same marker. (C) $2 \mathrm{D}$ electrophoresis of complex nucleic acid of sheared genomic DNA, comprising three populations of molecules of different sizes, i.e. interstrand crosslinked DNA, intact dsDNA and ssDNA. The populations were clearly separated. (D) Each fraction was size-determined using chromatogram and the relative amount of each fraction was calculated to be $28 \%, 53 \%$ and $19 \%$, respectively. (E) 2D electrophoresis of low-range ssRNA ladder (NEB) ranging from $50 \mathrm{nt}$ to $1000 \mathrm{nt}$. Size of RNA bands can be determined by comparing them to the single-stranded form of the $100 \mathrm{bp}$ DNA size marker placed directly below the RNA band.

The optimized electrode shape was an inverse-arrow shape at the tip to collect the gas bubbles for efficient evaporation from the gel into the vents and avoiding gas build-up within the cassette.

In the gel cassette design, there is no buffer outside the gel, making handling and sample loading easy. We tested the system by running samples with and without glycerol and Ficoll loading buffers. The results showed that one could easily avoid the use of loading buffers without compromising the electrophoresis.

We tested the system using various preparations of nucleic acids. As an example, we tested 1D electrophoresis using a 100 bp size standard and two different DNA fragments of 234 and $264 \mathrm{bp}$. Using 4\% acrylamide and a running time of 5 min gave excellent separation of bands (Figure 3A). To test the 2D electrophoresis, we first used Cy5-labelled dsDNA size marker. As can be seen in Figure 3B, the DNA fragments migrate as an arc in the gel as expected given the differential rate of migration of dsDNA and ssDNA molecules of different sizes. There was no indication of distortion in the electrical field during the second dimension and no indication of damage in the molecules of the size marker that affected their migration (in contrast to Figure 3C).

The 2D-SDE methodology (described above) can be used for analysis of the amount of sDNA and dsDNA, RNA-DNA hybrids, inter-strand crosslinked DNA and many other types of DNA damage. We tested if our apparatus could be used for 2D-SDE analysis and used samples that contained ssDNA, dsDNA and inter-strand crosslinked DNA. The system efficiently separated the three different fractions (Figure 3C). Using the ImageQuant ${ }^{\text {TM }}$ software we could determine the size distribution of each fraction (see chromatogram in Figure 3D) and measure the relative amount of each fraction.

The apparatus can also be used to analyze RNA. RNA samples separate according to length and as single-stranded molecules (Figure 3E). Ribosomal bands can be clearly seen in biological RNA samples and RNA can be distinguished from ssDNA by its RNase sensitivity and presence of ribosomal bands (data not shown).

Besides simple and quick 1D PAGE, 2D applications include characterization of efficiency of complex molecular procedures, checking quality of biosamples and detecting DNA damage in cells and body fluids. The system can run 1D electrophoresis of nucleic acids in 5 min with excellent separation making it an ideal system for use in teaching and research laboratories. For 2D electrophoresis, such as 2D-SDE, the sample can be electrophorized, poststained and scanned within a time span of $30 \mathrm{~min}$. Using poststaining with Ribogreen allowed use of as little as 250 pg for single bands and $1 \mathrm{ng}$ of a complex nucleic acid sample. To date, we have successfully run around 3000 gels on a single instrument. The system should also run SDS-PAGE and other protein gels.

\section{Author contributions}

HGT, GHG, PE, and JJJ conceived the project. HGT, GHG, and BG executed experiments, analyzed results, designed the gel cassette, and instrument, KL did the electric field calculations and designed the electrode arrangement. HGT and JJJ wrote the manuscript. PE and JJJ supervised the project.

\section{Acknowledgements}

We thank Brynja $\mathrm{H}$. Thorsteinsdottir for Figure 3B, which was included in her diploma thesis. Wideblue Ltd, advised on cassette design, material, and circuit board.

\section{Financial \& competing interests disclosure}

BG, GHG, HGT, and JJJ are stockholders in Lifeind ehf. and BioCule (Scotland) Ltd, companies that hold the patents to 2D-SDE and 2D PAGE microgels and apparatus respectively and are marketing the technology. HGT is employed by Lifeind ehf. Funding from SMART grant to BioCule (Scotland) Ltd from the Scottish Government and Icelandic Technology Development Fund grants 061363006 and 081202008 to Lifeind ehf. The authors have no other relevant affiliations or financial involvement 
with any organization or entity with a financial interest in or financial conflict with the subject matter or materials discussed in the manuscript apart from those disclosed.

No writing assistance was utilized in the production of this manuscript.

\section{Open access}

This work is licensed under the Creative Commons Attribution 4.0 License. To view a copy of this license, visit http://creativecommons.org/licenses/by/4.0/

\section{References}

1. Cohen AS, Najarian D, Smith JA, Karger BL. Rapid separation of DNA restriction fragments using capillary electrophoresis. $J$. Chromatogr. 458, 323-333 (1988).

2. Friedrich $\mathrm{SM}, \mathrm{Zec} \mathrm{HC}$, Wang TH. Analysis of single nucleic acid molecules in micro- and nano-fluidics. Lab Chip 16(5), 790-811 (2016).

3. Li T, Joshi MD, Ronning DR, Anderson JL. Ionic liquids as solvents for in situ dispersive liquid- liquid microextraction of DNA. J. Chromatogr. A 1272, 8-14 (2013).

4. Breeze DC, Thorne HV. Electrophoretic separation of two plaque-type variants of encephalomyocarditis virus. Nature 210(5040), 1080-1081 (1966).

5. Boulikas T, Hancock R. A highly sensitive technique for staining DNA and RNA in polyacrylamide gels using silver. J. Biochem. Biophys. Methods 5(4), 219-228 (1981).

6. Aaij C, Borst P. The gel electrophoresis of DNA. Biochim. Biophys. Acta 269(2), 192-200 (1972).

7. Consden R, Gordon AH, Martin AJ. Ionophoresis in silica jelly; a method for the separation of amino-acids and peptides. Biochem. J. 40(1), 33-41 (1946).

8. Gunnarsson GH, Gudmundsson B, Thormar $H G$, Alfredsson A, Jonsson JJ. Two-dimensional strandness-dependent electrophoresis. Nat. Protoc. 1(6), 3011-3018 (2006).

9. Gunnarsson GH, Gudmundsson B, Thormar $H G$, Alfredsson A, Jonsson JJ. Two-dimensional strandness-dependent electrophoresis: a method to characterize single-stranded DNA, double-stranded DNA, and RNA-DNA hybrids in complex samples. Anal. Biochem. 350(1), 120127 (2006).

10. Glavac D, Dean M. Heteroduplex analysis. In: Technologies for Detection of DNA Damage and
Mutations, Pfeifer $\mathrm{G}$ (Ed.). Plenum Press, New York, USA, 241-249 (1996).

11. Fischer SG, Lerman LS. Length-independent separation of DNA restriction fragments in two-dimensional gel electrophoresis. Cell 16(1), 191-200 (1979).

12. Rosenbaum V, Riesner D. Temperature-gradient gel electrophoresis. Thermodynamic analysis of nucleic acids and proteins in purified form and in cellular extracts. Biophys. Chem. 26(2-3), 235-246 (1987).

13. Biocule (Scotland) Limited. WO2012107774 (2012).

First draft submitted: 31 March 2017; Accepted for publication: 17 May 2018

Address correspondence to: Jon J Jonsson; Department of Biochemistry \& Molecular Biology, Faculty of Medicine, University of Iceland, Reykjavik, Iceland; Tel.: +354 824 5917; E-mail: jonjj@hi.is

To purchase reprints of this article contact: s.cavana@future-science.com

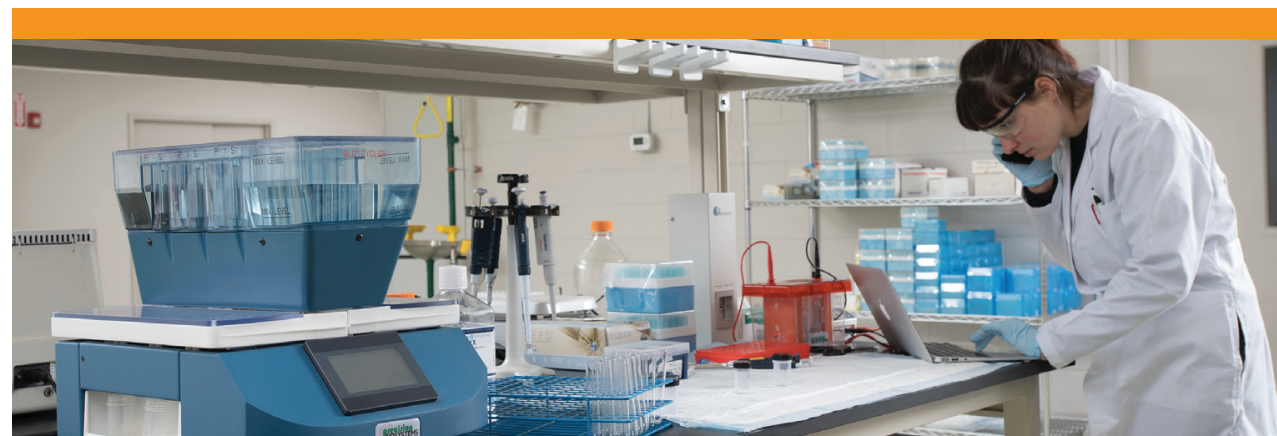

\section{Blot-to-blot consistency. Time after time.}

To achieve meaningful comparison of expressed proteins from blot-to-blot, trustworthy data is essential. BlotCycler $^{\mathrm{TM}}$ is designed to eliminate slight variations in fluid delivery, timing and washing that may occur when protocols are performed

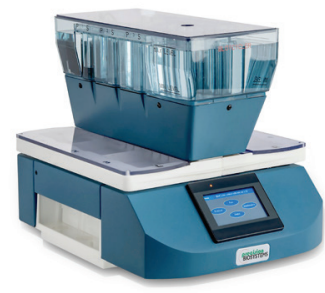
manually. With rigorous precision, BlotCycler delivers an affordable solution to busy labs demanding reproducible results.

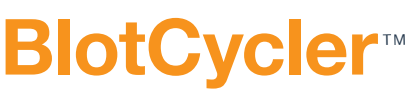

bY PRECISION BIOSYSTEMS

www.blotcycler.com 